\title{
Regulatory role of microRNA-30b and plasminogen activator inhibitor-1 in the pathogenesis of cognitive impairment
}

\author{
XIUQIN LI ${ }^{1}$, YONG GAO ${ }^{2}$, ZHAOYUN MENG $^{1}, \mathrm{CUI} \mathrm{ZHANG}^{3}$ and QINDE QI $^{3}$ \\ ${ }^{1}$ Second Department of Health; Departments of ${ }^{2}$ Neurosurgery and ${ }^{3}$ Neurology, \\ Laiwu Hospital Affiliated to Taishan Medical University, Laiwu, Shandong 271100, P.R. China
}

Received May 18, 2015; Accepted January 15, 2016

DOI: $10.3892 /$ etm.2016.3162

\begin{abstract}
The present study aimed to investigate the role of plasminogen activator inhibitor-1 (PAI-1) in drug-induced early cognitive impairment and the underlying mechanism concerning microRNA (miR)-30b. A mouse model of cognitive impairment was established by intraperitoneal injection of scopolamine ( $2 \mathrm{mg} / \mathrm{kg}$ body weight) for 13 days. Behavioral performance was assessed using the Morris water maze (MWM) test. The mRNA expression levels of PAI-1 and miR-30b were detected using quantitative polymerase chain reaction (qPCR). The protein expression levels of PAI-1 in the hippocampus and blood were determined using western blot analysis and enzyme-linked immunosorbent assays. The MWM test demonstrated that, on days 3 and 4, the escape latency was significantly elevated in the model mice in comparison with control group $(\mathrm{P}<0.05)$. In addition, the length of swimming path was significantly increased $(\mathrm{P}<0.05)$, while the number of times of crossing the platform location was significantly reduced in the model mouse group $(\mathrm{P}<0.05)$ in comparison with the control group. qPCR demonstrated that the mRNA expression levels of PAI-1 in the model mice was significantly elevated in the hippocampus and blood in comparison with the control group $(\mathrm{P}<0.01)$. Furthermore, western blot analysis and enzyme-linked immunosorbent assay demonstrated that the protein expression levels of PAI-1 were significantly elevated in the hippocampus and blood in the model group, in comparison with the control group $(\mathrm{P}<0.05)$. Notably, the levels of miR-30b in the hippocampus and blood were significantly decreased in the model mice in comparison with the control group $(\mathrm{P}<0.01)$. To conclude, the expression levels of PAI-1 were significantly elevated in mice with scopolamine-induced cognitive impairment, which may be associated with the downregulation of $\mathrm{miR}-30 \mathrm{~b}$. The findings from the present study suggest that miR-30b may be involved in
\end{abstract}

Correspondence to: Dr Qinde Qi, Department of Neurology, Laiwu Hospital Affiliated to Taishan Medical University, 1 Xuehu Avenue, Laiwu, Shandong 271100, P.R. China

E-mail: qqd999@126.com

Key words: microRNA-30b, cognitive impairment, plasminogen activator inhibitor-1 the regulation of PAI-1, which would contribute to the pathogenesis of cognitive impairment.

\section{Introduction}

Cognitive impairment is a common manifestation of a variety of neurological disorders and is characterized by an impairment of learning and memory function (1). The pathological process of cognitive impairment is accompanied by aphasia, apraxia, agnosia and a number of other changes in behavioral performance (2). Cognitive impairment may develop into Alzheimer's disease, which detrimentally affects the quality of life of patients, and increases the burden on their family and society (1). At present, a clinical diagnosis of cognitive impairment relies on neuropsychological tests, blood tests, neuroimaging, electroencephalography and cerebrospinal fluid tests (3). The pathogenesis of cognitive impairment has yet to be fully elucidated (4). Several widely accepted pathological causes of the disease include the cerebral ischemia, hypoxia and neuronal necrosis in brain tissue caused by trauma, cerebral vascular inflammation, vascular stenosis and brain embolism (5). In particular, research concerning the disease pathogenesis has focused on vascular lesions in the brain (6).

Vascular diseases of the brain may be caused by an abnormality in the blood rheology that may also be involved in the pathological process of other diseases (7). Plasminogen activator inhibitor-1 (PAI-1) is a serine protease inhibitor that inactivates the plasminogen activators t-PA and u-PA, and inhibits intravascular fibrinolysis that results in further changes in blood rheology and an increased risk of ischemic injury (8). PAI-1 has been recognized as a risk factor for the pathogenesis and development of ischemic cerebrovascular and cardiovascular diseases $(9,10)$. It has been demonstrated that PAI-1 is targeted by micro RNA (miR)-30b to regulate the proliferation and apoptosis of gastric cancer cells (11). However, the role of PAI-1 and its upstream regulators in cognitive impairment has not been fully established.

In the present study, a mouse model of cognitive impairment was induced by scopolamine, and the role of PAI-1 in the disease pathogenesis was investigated. Behavioral performance tests were performed and assessed by the Morris water maze (MWM) test. The mRNA and protein expression levels of PAI-1 were determined, and the underlying mechanism concerning miR-30b was explored. 


\section{Materials and methods}

Animal model. A total of 80 male Kunming mice (weight, 18-22 g; age, 4 weeks), were purchased from Xinteng Bier Animal Co., Ltd. (SCXK 2012-0008; Chongqing, China) and housed in cages at $22^{\circ} \mathrm{C}, 56 \%$ humidity with a $12 \mathrm{~h}$ light/dark cycle and ad libitum access to food and water. Each animal experimental procedure was approved by the Care of Experimental Animals Committee of Laiwu Hospital Affiliated to Taishan Medical University (Laiwu, China). The animal cognitive impairment model was established by daily intraperitoneal injection of scopolamine hydrobromide for 13 days ( $2 \mathrm{mg} / \mathrm{kg}$ body weight; H19994038; Furen Medicines Group, Henan, China) for 13 days. The normal control group was injected with saline.

MWM test. The behavioral performance of the model mice was assessed using the MWM test (Institute of Materia Medica, Chinese Academy of Medical Science and Peking Union Medical College, Beijing, China). The test commenced following the 13 day period of scopolamine hydrobromide injections. The navigation test was performed for 4 days, and each mouse was subjected to 4 tests daily. The swimming pool was $0.8 \mathrm{~m}$ in diameter and divided into four quadrants of equal size. The water was $30 \mathrm{~cm}$ in depth and a hidden platform was placed $1 \mathrm{~cm}$ under the surface in the center of one quadrant. A cardinal point of the quadrant without the platform was randomly selected as the start location. The test commenced when a mouse was placed in the pool, and ended when the mouse located the platform. The escape latency was recorded as time that the mouse spent searching for the platform. If a mouse failed to locate the platform within $60 \mathrm{sec}$, the escape latency was recorded as $60 \mathrm{sec}$ and the mouse was guided to the platform. After remaining on the platform for $15 \mathrm{sec}$, the next test began. On day 5 the probe test was performed, in which the platform was removed and each mouse was allowed to swim freely in the pool for $60 \mathrm{sec}$. The swimming path length, as well as the number of times that each mouse swam across the region where the platform used to be, was recorded.

Tissue sample preparation. Blood samples were obtained following the behavioral test. The mice were fasted for $12 \mathrm{~h}$ and anesthetized by intraperitoneal injection of $10 \%$ chloral hydrate (0.0004, 1/g body weight; Sinopharm Chemical Reagent Co., Ltd., Shanghai, China). Abdominal aortic blood was collected and the mice were sacrificed by cervical dislocation. The serum was separated and stored at $-80^{\circ} \mathrm{C}$. The mouse brains were removed and the hippocampus was separated. The hippocampal tissue was washed with $0.9 \%$ cold saline and stored at $-80^{\circ} \mathrm{C}$.

Reverse transcription-quantitative polymerase chain reaction (RT-qPCR). The mRNA levels of PAI-1 and the levels of miRNA-30b were detected in the blood samples using qPCR. Total RNA ( $2 \mu \mathrm{l})$ from the hippocampus was extracted using TRIzol reagent (Yeasen Corporation, Shanghai, China) and RNA in the blood samples was extracted using the miRNeasy SerumPlasma kit (Guangzhou Jianlun Biological Technology Co., Ltd., Guangzhou, China). Residual genomic DNA was removed using DNase (Cloud-Clone Corporation,
Houston, TX, USA) Reverse transcription was performed to obtain cDNA using the miRcute miRNA cDNA First-strand Synthesis kit [Tiangen Biotech (Beijing) Co., Ltd., Beijing, China]. qPCR was performed using the miRcute miRNA Quantitative Fluorescence Detection kit [Tiangen Biotech (Beijing) Co., Ltd.] and PCR-iQ5 Multicolor Real-time PCR Detection System (Bio-Rad Laboratories, Inc., Hercules, CA, USA). The $25 \mu$ l PCR system consisted of $12.5 \mu 1$ SuperReal PreMix [Tiangen Biotech (Beijing) Co., Ltd.], $1 \mu \mathrm{l}$ primer, $2 \mu \mathrm{l}$ cDNA template and $5 \mu \mathrm{l} \mathrm{dd}_{2} \mathrm{O}$. For PAI-1 detection, the primer sequences were as follows: PAI-1 forward, 5'-TCT CCGCCATCACCAACATT-3' and reverse, 5'-GAGAGA ACTTAGGCAGGATGAGG-3'; $\beta$-actin forward, 5'-AAC CCTAAGGCCAACAGTGAAAAG-3' and reverse, 5'-TCA TGAGGTAGTCTGTGAGGT-3'. The reaction conditions consisted of denaturation at $95^{\circ} \mathrm{C}$ for $2 \mathrm{~min}, 95^{\circ} \mathrm{C}$ for $30 \mathrm{sec}$, $58^{\circ} \mathrm{C}$ for $30 \mathrm{sec}$ and $72^{\circ} \mathrm{C}$ for $30 \mathrm{sec}$ for a total of 40 cycles. For miR-30b detection, the primer sequences were as follows: miR-30b forward, 5'-GCGCCTGTAAACATCCTACAC-3' and reverse, 5'-GTGCAGGGTCCGAGGT-3'; U6 forward, 5'-GCTTCGGCAGCACATATACTAA-3' and reverse, 5'AAC GCTTCACGAATTTGCGT-3'. Primers were synthesized by Sangon Biotech Co., Ltd. (Shanghai, China). The reaction conditions were $95^{\circ} \mathrm{C}$ for $30 \mathrm{sec}, 95^{\circ} \mathrm{C}$ for $5 \mathrm{sec}$ and $60^{\circ} \mathrm{C}$ for $30 \mathrm{sec}$ for a total of 45 cycles. Three repeats were performed of each sample, without a negative and RT control. The relative expression levels of the target genes were calculated using the $2^{-\Delta \Delta C q}$ method (12). The results were analyzed using Image Lab software (version 3.0; Bio-Rad Laboratories, Inc.).

Western blot analysis. The protein expression levels of PAI-1 in the hippocampal tissues were detected using western blot analysis. Tissues were lysed with lysis buffer (Beyotime Institute of Biotechnology, Haimen, China) and the protein concentration was determined using a bicinchoninic acid assay kit (RTP7102; Real-Times Biotechnology Co., Ltd., Beijing, China). Each $20 \mu \mathrm{g}$ protein sample was subjected to $10 \%$ sodium dodecyl sulfate polyacrylamide gel electrophoresis (Wuhan Boster Biological Technology, Ltd., Wuhan, China) and electronically transferred onto a polyvinylidene fluoride membrane (Shanghai Yuanye Biotechnology Co. Ltd., Shanghai, China). The membrane was blocked with 5\% fat-free milk at room temperature for $1 \mathrm{~h}$ and then incubated with rabbit anti-mouse anti-PAI-1 primary antibody $(1: 1,000$; ab66705; Abcam, Cambridge, MA, USA), or rabbit anti-mouse anti- $\beta$-actin antibody $\left(1: 5,000\right.$; ab129348; Abcam) at $4^{\circ} \mathrm{C}$ overnight. The membrane was then incubated with goat anti-rabbit horseradish peroxidase (HRP)-conjugated secondary antibody $(1: 3,000 ;$ ab6721; Abcam) at room temperature for $1 \mathrm{~h}$. The protein bands were visualized using an enhanced chemiluminescence kit (ab65623; Abcam) and analyzed using Image Lab software (version 3.0). $\beta$-actin was used as the internal control.

Enzyme-linked immunosorbent assay (ELISA). The protein levels of PAI-1 in the blood samples were determined using an ELISA kit (Abcam). Blood samples were centrifuged at $1,500 \mathrm{rpm}$ for $10 \mathrm{~min}$ to separate the serum from the red blood cells. A $10 \mu \mathrm{l}$ sample and $40 \mu \mathrm{l}$ dilution solution was added into each well on the microplate. Next, $100 \mu$ l HRP-labeled detection antibody was added and the plate was sealed and 
A

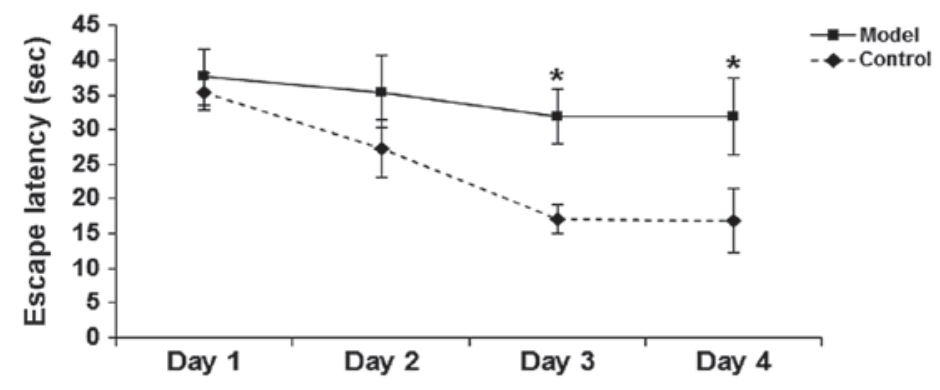

B

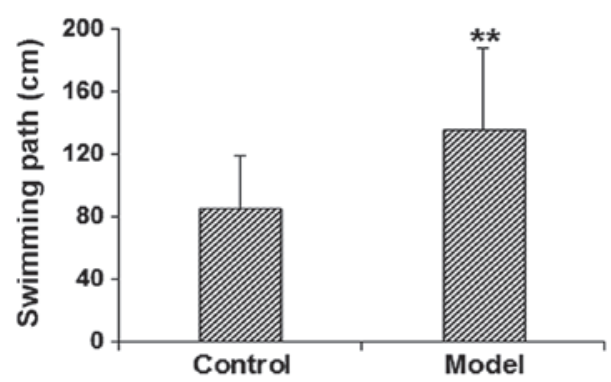

C

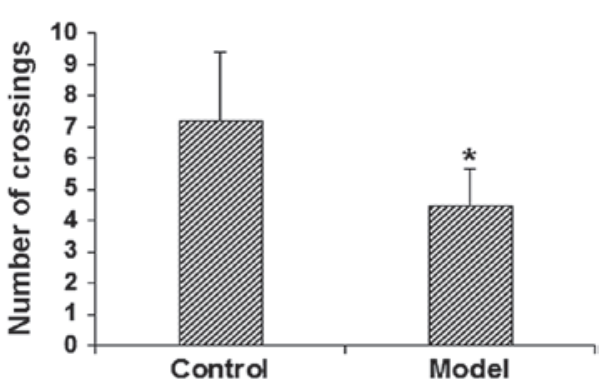

Figure 1. Declined learning and memory function of scopolamine-treated mice. The behavioral performance was assessed using the Morris water maze test. (A) The escape latency and the probe test evaluating (B) the length of swimming path and (C) the times of crossing the platform location. ${ }^{*} \mathrm{P}<0.05,{ }^{* *} \mathrm{P}<0.01$ vs. the control group.

incubated for $1 \mathrm{~h}$ at $37^{\circ} \mathrm{C}$, followed by washing with washing buffer included in the kit. After washing for 5 times, $50 \mu \mathrm{l}$ substrate A and $50 \mu \mathrm{l}$ substrate $\mathrm{B}$ was added into each well and the plate was incubated at $37^{\circ} \mathrm{C}$ for $15 \mathrm{~min}$. Finally, $50 \mu \mathrm{l}$ stop solution was added to stop the reaction and the optical density was read at $450 \mathrm{~nm}$ using a microplate reader (Bio-Rab Laboratories, Inc.) within $15 \mathrm{~min}$. The standard concentration curve was obtained using the standard samples provided with the ELISA kit (ab108891; Abcam).

Bioinformatics. Bioinformatic analysis was performed using the online target gene prediction tools, including miRanda (http://www.microrna.org/microrna/home.do), TargetScan (www.targetscan.org), PITA (http:/genie.weiz mann.ac.il/pubs/mir07/mir07_data.html), RNAhybrid (http://bibiserv.techfak.uni-bielefeld.de/rnahybrid/) and PICTA (http://pictar.mdc-berlin.de/).

Statistical analysis. Data is expressed as the mean \pm standard error. SPSS software, version 18.0 (SPSS, Inc., Chicago, IL, USA) was used to perform the statistical analysis. Data was subjected to the normality test. One-way analysis of variance was performed for multiple comparisons. The Least Significant Difference and Student-Newman-Keuls tests were performed for equal variance, and the Tamhane's T2 and Dunnett's T3 tests were performed when equal variance was not assumed. $\mathrm{P}<0.05$ was considered to indicate a statistically significant difference.

\section{Results}

Learning and memory function is reduced in model mice. To assess the learning and memory function of the model mice, the MWM test was performed. The results demonstrated that on days $3-4$, the escape latency was significantly elevated in the model group in comparison with the control group $(\mathrm{P}<0.05$; Fig. 1A). In addition, the probe test on day 5 indicated that, compared with the control group, the length of swimming path was significantly increased $(\mathrm{P}<0.05$; Fig. $1 \mathrm{~B})$, while the number of times of crossing the platform location was significantly reduced $(\mathrm{P}<0.05$; Fig. $1 \mathrm{C})$ in the model group. These results suggest that the learning and memory function is significantly impaired in the scopolamine-induced model mice.

PAI-1 expression levels are increased in the hippocampus and blood of model mice. To investigate the role of PAI-1 in the pathogenesis of cognitive impairment, the mRNA and protein expression levels of PAI-1 in the hippocampus and blood were detected. The mRNA expression level of PAI-1 was determined using RT-qPCR. The results demonstrated that, in comparison with the control group, the mRNA expression levels of PAI-1 in the model group were significantly elevated in the hippocampus and blood $(\mathrm{P}<0.01$; Fig. $2 \mathrm{~A}$ and $\mathrm{B})$. The protein expression levels of PAI-1 in the hippocampus and blood were evaluated using western blot analysis and ELISA. The results from the western blot analysis demonstrated that the protein expression level of hippocampal PAI-1 was significantly elevated in the model group in comparison with the control group $(\mathrm{P}<0.05$; Fig. $2 \mathrm{C})$. In addition, the results from the ELISA demonstrated that, in comparison with the control group, the level of PAI-1 in the blood was significantly increased in the model group $(\mathrm{P}<0.01$; Fig. 2D). These results suggest that PAI-1 may play a regulatory role in the pathogenesis of scopolamine-induced cognitive impairment.

miR-30b levels are decreased in the hippocampus and blood of model mice. It has been reported that PAI-1 may be regulated by $\mathrm{miR}-30 \mathrm{~b}$ in gastric cancer (11). According to the bioinformatic analysis using miRanda, TargetScan, PITA, RNAhybrid and PICTA software, it was concluded that miR-30b may be 
A
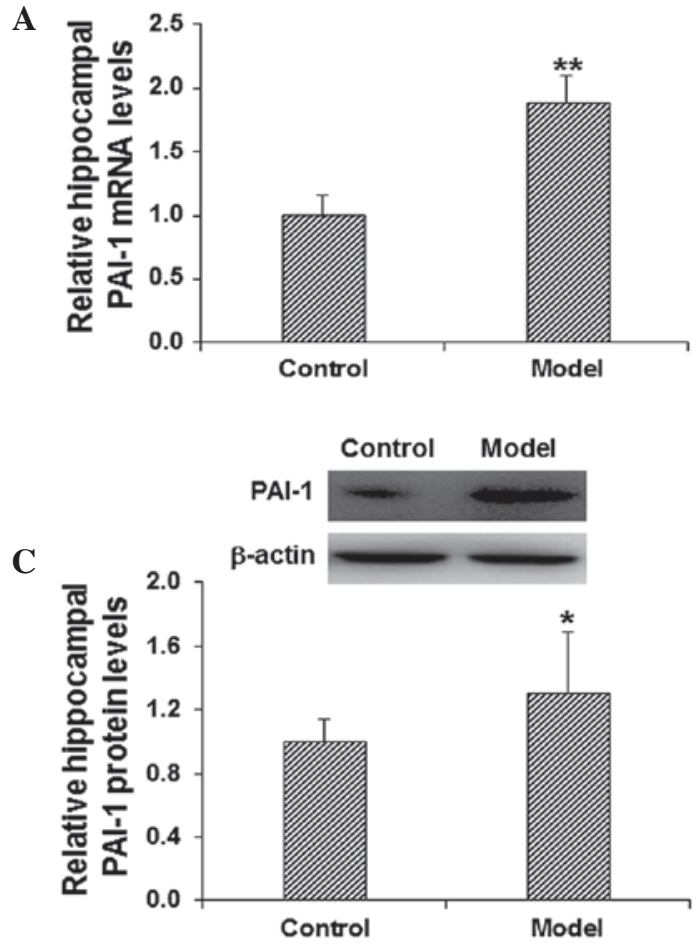

B

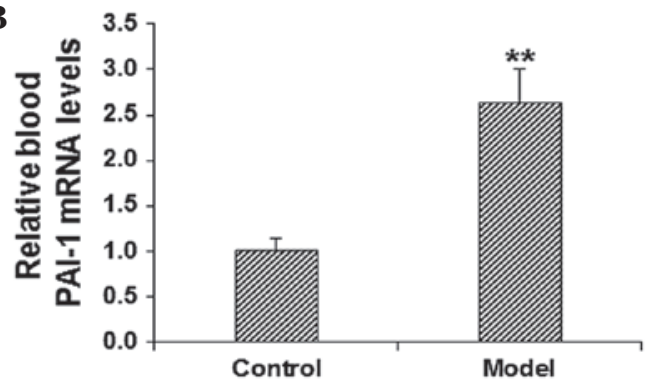

D

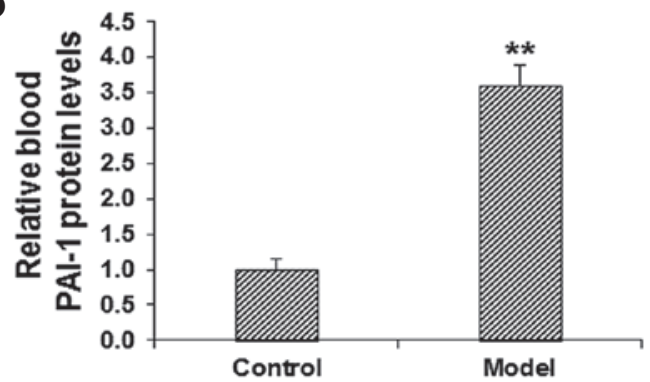

Figure 2. Increased PAI-1 expression levels in the hippocampal and blood tissues in model mice. The mRNA expression levels of PAI-1 in the (A) hippocampus and (B) blood were determined by quantitative polymerase chain reaction. The protein expression levels of PAI-1 in the (C) hippocampus and (D) blood were detected using western blot analysis and enzyme-linked immunosorbent assay. ${ }^{*} \mathrm{P}<0.05,{ }^{* *} \mathrm{P}<0.01$ vs. the control group. PAI-1, plasminogen activator inhibitor-1.

\section{3' UCGACUCACAUC-----CU-----ACAAAUGu 5' mmu-miR-30b I IIIIIIII II IIIIIII 555:5' UuCAGAGUGUAGGUGACUUGUUUACa 3' Serpinel}

Figure 3. Bioinformatic analysis of the upstream regulators of plasminogen activator inhibitor-1. miR, microRNA.

a regulator of PAI-1 (Fig. 3). To further confirm the role of miR-30b in the regulation of PAI-1 in cognitive impairment, the mRNA expression levels of miR-30b in the hippocampus and blood in scopolamine-treated model mice were detected using RT-qPCR. The results demonstrated that, in comparison with the control group, the level of miR-30b in the hippocampus and blood was significantly decreased in the model group ( $\mathrm{P}<0.01$; Fig. 4). These results suggest that miR-30b may be involved in the pathogenesis and development of cognitive impairment.

\section{Discussion}

Cognitive impairment is a chronic central nervous system disorder that is commonly observed in the elderly population (13). Cognitive impairment is a pathological state that, without treatment, may gradually develop into different forms of dementia, such as Alzheimer's disease (14). At present, blood tests are primarily used to identify concomitant diseases, complications and potential risks. Cerebrospinal fluid examination is characterized by high sensitivity and specificity in the detection of disease; however, due to the lack of standardized procedures for sample collection and preparation, diagnosis based on blood tests or cerebrospinal fluid examination alone
A

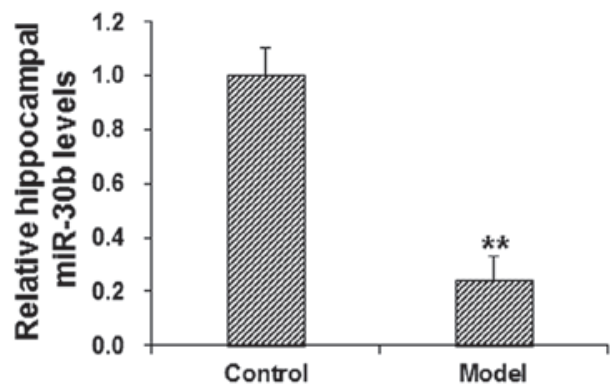

B

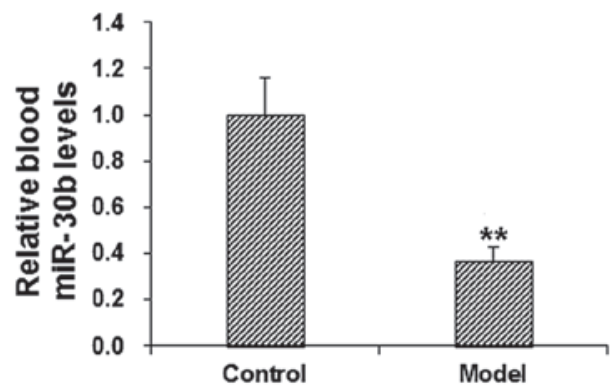

Figure 4. Decreased miR-30b levels in the hippocampus and blood of model mice. The levels of miR-30b in the (A) hippocampal and (B) blood tissues were determined by the reverse transcription-quantitative polymerase chain reaction. ${ }^{* *} \mathrm{P}<0.01$ vs. the control group. miR, microRNA.

may result in misdiagnosis and/or missed (15). Therefore, there is an urgent requirement for further stable genetic markers for the diagnosis and the treatment of cognitive impairment.

The human brain is an extremely complex organ in which the cerebral cortex forms the foundation of cognitive function; therefore, an abnormality in the structure and function of the cerebral cortex may result in cognitive impairment (16). 
Research into cognitive function typically focuses on the hippocampus, in particular the hippocampal neurons that serve a key role in cognitive regulation (17).

In the present study, a mouse model of cognitive impairment was established using scopolamine (18), and the learning and memory function of these mice was evaluated using the MWM test. Scopolamine is a muscarinic receptor antagonist that specifically binds to, and inhibits, muscarinic M1 receptors distributed in the hippocampus and neocortex (19). It is understood that scopolamine prevents the trophic and protective effects of M1 receptor antagonists on neurons, inhibits neurite growth, promotes $\beta$-amyloid peptide-induced cell apoptosis, increases the phosphorylation of Tau protein (20) and causes a decline in memory $(21,22)$.

The results from the MWM test in the present study demonstrated that the escape latency was significantly elongated in model mice in comparison with control mice. In addition, the probe test demonstrated that in model mice the navigation path was significantly longer, and the number of times crossing the platform region was significantly reduced. These results suggest that the learning and memory function is markedly impaired in scopolamine-treated mice, therefore identifying them as a suitable model of cognitive impairment.

One of the pathological features of cognitive impairment is the vascular amyloid deposition in cortical arteries and arterioles, which is closely linked with PAI-1 (23). It has been demonstrated that PAI-1 is able to regulate the formation of thrombosis, and that it is involved in the accumulation of extracellular matrix and the migration of smooth muscle cells (24-28). Furthermore, PAI-1 has been observed to induce the deposition of low density lipoprotein within the extracellular matrix of vascular smooth muscle cells, promote the formation of fatty streaks and atherosclerotic plaques, and thicken the vascular basement membrane (29). These pathological changes may result in vascular diseases and induce the occurrence and development of atherosclerosis (24-28).

In the present study the results demonstrated that, in comparison with the control group, the mRNA and protein expression levels of PAI-1 were significantly elevated in the hippocampus and blood of the model group, suggesting that PAI-1 may serve a regulatory role in the development of cognitive impairment. It is widely accepted that PAI-1 is closely associated with vascular lesions, which would cause abnormal blood flow and other pathological changes including cerebral ischemia, hypoxia, metabolite accumulation and cognitive impairment $(30,31)$. Therefore, it is important to investigate the mechanisms by which PAI-1 is regulated in disease pathogenesis.

miRs are a class of endogenous, small, non-coding RNAs that modulate gene expression via the negative regulation of target mRNA $(32,33)$. miRs are important regulators under physiological and/or pathological conditions, and have become biomarkers for various pathological changes $(34,35)$. Using bioinformatic analysis in the current study, the upstream regulatory genes of PAI-1 were predicted; miR-30b was identified as a regulatory gene of PAI-1, and this is in accordance with a previous study (11). Furthermore, miR-30b has been observed to be associated with the pathogenesis of schizophrenia (36). According to the results in the present study, the levels of miR-30b were significantly downregulated in scopolamine-induced cognitive impairment, in contrast with the upregulation of the mRNA and protein expression levels of PAI-1. These results are in accordance with previous findings by Zhu et al (11), indicating that the downregulation of miR-30b may be involved in the early onset and development of cognitive impairment. Based on these findings, it can be hypothesized that the change in expression levels of miR-30b in blood can be used as a potential indicator for the diagnosis of cognitive impairment, and may be applied in the clinical treatment of the disease in the future.

In conclusion, the results from the current study demonstrate that in scopolamine-induced mouse models of cognitive impairment, the mRNA and protein expression levels of PAI-1 are significantly elevated, while the miR-30b levels are significantly declined, in the hippocampus and blood. These pathological changes may be associated with the apoptotic processes and the vascular lesions that can ultimately result in cognitive impairment. These findings suggest that miR-30b is a potent regulator of PAI-1 in cognitive impairment, and may have the potential to be used in the diagnosis and treatment of cognitive impairment in the future.

\section{Acknowledgements}

The authors thank Director Fangyu Song from the Department of Neurology, Laiwu Hospital Affiliated to Taishan Medical University (Laiwu, China) for assistance with the experimental design and performance, data collection and analysis and manuscript preparation.

\section{References}

1. Shen X, Li Y and Xu L: Correlation between arterial microemboli and vascular cognitive impairment in patients with acute cerebral infarction. Zhong Guo Lao Nian Xue Za Zhi She 33: 1400-1402, 2013 (In Chinese)

2. Miura R and Hattori H: Overview and assessment of cognitive function in interpreting postoperative cognitive dysfunction. Masui 63: 1188-1195, 2014 (In Japanese).

3. Li S, Okonkwo O, Albert M and Wang MC: Variation in Variables that Predict Progression from MCI to AD Dementia over Duration of Follow-up. Am J Alzheimers Dis (Columbia) 2: 12-28, 2013.

4. Petrella JR: Neuroimaging and the search for a cure for Alzheimer disease. Radiology 269: 671-691, 2013.

5. de la Monte SM and Tong M: Brain metabolic dysfunction at the core of Alzheimer's disease. Biochem Pharmacol 88: 548-559, 2014.

6. Banerjee G, Wilson D, Jäger HR and Werring DJ: Novel imaging techniques in cerebral small vessel diseases and vascular cognitive impairment. Biochim Biophys Acta: 10 Dec, 2015 (Epub ahead of print).

7. Anisimova AV, Kolesnikova TI, Iutskova EV, Galkin SS and Zimin IA: An impact of neuroprotective therapy on blood rheological and morphodensitometric parameters in patients with chronic cerebral ischemia. Zh Nevrol Psikhiatr Im S S Korsakova 114: 72-80, 2014 (in Russian).

8. Hua Y, Xi G, Keep RF, Wu J, Jiang Y and Hoff JT: Plasminogen activator inhibitor-1 induction after experimental intracerebral hemorrhage. J Cereb Blood Flow Metab 22: 55-61, 2002.

9. Kohler HP and Grant PJ: Plasminogen-activator inhibitor type 1 and coronary artery disease. N Engl J Med 342: 1792-1801, 2000.

10. Eitzman DT, Westrick RJ, Xu Z, Tyson J and Ginsburg D: Plasminogen activator inhibitor-1 deficiency protects against atherosclerosis progression in the mouse carotid artery. Blood 96: 4212-4215, 2000.

11. Zhu ED, Li N, Li BS, Li W, Zhang WJ, Mao XH, Guo G, Zou QM and Xiao B: miR-30b, down-regulated in gastric cancer, promotes apoptosis and suppresses tumor growth by targeting plasminogen activator inhibitor-1. PLoS One 9: e106049, 2014. 
12. Livak KJ and Schmittgen TD: Analysis of relative gene expression data using real-tie quantitative PCR and the 2(-Delta Delta C(T)) Method. Methods 25: 402-408, 2001.

13. Hildreth KL and Church S: Evaluation and management of the elderly patient presenting with cognitive complaints. Med Clin North Am 99: 311-335, 2015.

14. Kapasi A and Schneider JA: Vascular contributions to cognitive impairment, clinical Alzheimer's disease, and dementia in older persons. Biochim Biophys Acta: 5 Jan, 2016 (Epub ahead of print).

15. Mishra D, Sharma S, Gupta S, Das M and Chauhan D: Acute cysticercal meningitis - missed diagnosis. Indian J Pediatr 73: 835-837, 2006.

16. Armato U, Chakravarthy B, Pacchiana R and Whitfield JF: Alzheimer's disease: An update of the roles of receptors, astrocytes and primary cilia. Int J Mol Med 31: 3-10, 2013.

17. Christie BR and Cameron HA: Neurogenesis in the adult hippocampus. Hippocampus 16: 199-207, 2006.

18. Ikonomovic MD, Abrahamson EE, Isanski BA, Wuu J, Mufson EJ and DeKosky ST: Superior frontal cortex cholinergic axon density in mild cognitive impairment and early Alzheimer disease. Arch Neurol 64: 1312-1317, 2007.

19. Ortega A, del Guante MA, Prado-Alcalá RA and Alemán V: Changes in rat brain muscarinic receptors after inhibitory avoidance learning. Life Sci 58: 799-809, 1996.

20. Jean L, Thomas B, Tahiri-Alaoui A, Shaw M and Vaux DJ: Heterologous amyloid seeding: Revisiting the role of acetylcholinesterase in Alzheimer's disease. PLoS One 2: e652-e660, 2007.

21. Geula C, Nagykery N, Nicholas A and Wu CK: Cholinergic neuronal and axonal abnormalities are present early in aging and in Alzheimer disease. J Neuropathol Exp Neurol 67: 309-318, 2008.

22. Minger SL, Esiri MM, McDonald B, Keene J, Carter J, Hope T and Francis PT: Cholinergic deficits contribute to behavioral disturbance in patients with dementia. Neurology 55: 1460-1467, 2000.

23. Erren M, Reinecke H, Junker R, Fobker M, Schulte H, Schurek JO, Kropf J, Kerber S, Breithardt G, Assmann G and Cullen P: Systemic inflammatory parameters in patients with atherosclerosis of the coronary and peripheral arteries. Arterioscler Thromb Vasc Biol 19: 2355-2363, 1999.

24. Hamsten A, de Faire U, Walldius G, Dahlén G, Szamosi A, Landou C, Blombäck M and Wiman B: Plasminogen activator inhibitor in plasma: Risk factor for recurrent myocardial infarction. Lancet 2: 3-9, 1987.
25. Thögersen AM, Jansson JH, Boman K, Nilsson TK, Weinehall L, Huhtasaari F and Hallmans G: High plasminogen activator inhibitor and tissue plasminogen activator levels in plasma precede a first acute myocardial infarction in both men and women: Evidence for the fibrinolytic system as an independent primary risk factor. Circulation 98: 2241-2247, 1998.

26. Lupu F, Bergonzelli GE, Heim DA, Cousin E, Genton CY, Bachmann F and Kruithof EK: Localization and production of plasminogen activator inhibitor-1 in human healthy and atherosclerotic arteries. Arterioscler Thromb 13: 1090-1100, 1993.

27. Lupu F, Heim DA, Bachmann F, Hurni M, Kakkar VV and Kruithof EK: Plasminogen activator expression in human atherosclerotic lesions. Arterioscler Thromb Vasc Biol 15: 1444-1455, 1995.

28. Olexa P and Olexová M: Plasminogen activator inhibitor-1 (PAI-1), ischemic heart disease and diabetes mellitus. Vnitr Lek 49: 222-226, 2003 (In Slovak).

29. Rouch A, Vanucci-Bacqué C, Bedos-Belval F, and Baltas M: Small molecules inhibitors of plasminogen activator inhibitor-1 - an overview. Eur J Med Chem 92: 619-636, 2015.

30. Jokinen H, Gonçalves N, Vigário R, Lipsanen J, Fazekas F, Schmidt R, Barkhof F, Madureira S, Verdelho A, Inzitari D, et al: Early-stage white matter lesions detected by multispectral MRI segmentation predict progressive cognitive decline. Front Neurosci 9: 455, 2015.

31. Matsuura Y, Yamashita A, Iwakiri T, Sugita C, Okuyama N, Kitamura $\mathrm{K}$ and Asada Y: Vascular wall hypoxia promotes arterial thrombus formation via augmentation of vascular thrombogenicity. Thromb Haemost 114: 158-172, 2015.

32. Inoue K: MicroRNA function in animal development. Tanpakushitsu Kakusan Koso 52: 197-204, 2007 (In Japanese).

33. Williams AE, Moschos SA, Perry MM, Barnes PJ and Lindsay MA: Maternally imprinted microRNAs are differentially expressed during mouse and human lung development. Dev Dyn 236: 572-580, 2007

34. Li X, Yu Z, Li Y, Liu S, Gao C, Hou X, Yao R and Cui L: The tumor suppressor miR-124 inhibits cell proliferation by targeting STAT3 and functions as a prognostic marker for postoperative NSCLC patients. Int J Oncol 46: 798-808, 2015.

35. Lv ZC, Fan YS, Chen HB and Zhao DW: Investigation of microRNA-155 as a serum diagnostic and prognostic biomarker for colorectal cancer. Tumour Biol 36: 1619-1625, 2015.

36. Mellios N, Galdzicka M, Ginns E, Baker SP, Rogaev E, Xu J and Akbarian S: Gender-specific reduction of estrogen-sensitive small RNA, miR-30b, in subjects with schizophrenia. Schizophr Bull 38: 433-443, 2012. 